KOMPASS

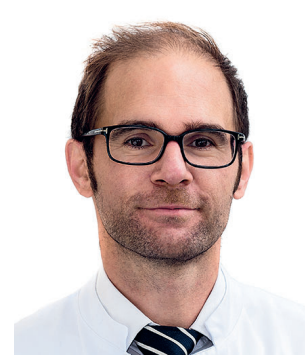

Andreas Körber

Gemeinschaftspraxis Dr. Cesko \& Prof. Dr. Körber, Essen, Deutschland

Liebe Kolleginnen und Kollegen, ich freue mich, in der vorliegenden Ausgabe des Karger KOMPASS DeRmatologie eine Vielzahl spannender und breit gefächerter Arbeiten vorstellen zu können. Wie gewohnt von ausgewählten Experten kommentiert und mit einem konkreten Fazit für die Praxis versehen. Grundthema dieser Ausgabe ist der alte Mensch, respektive Dermatologie im Alter. Alterung betrifft nahezu alle Organsysteme und zeigt sich isoliert oder im Zuge systemischer Prozesse an der Haut. Hauterkrankungen im Alter und die Altersdermatologie rücken in den letzten Jahren mehr und mehr in den Fokus, da die wissenschaftliche Grundlage zu einer Vielzahl von Alterungsprozessen an der Haut noch in den Kinderschuhen steckt. Neben den klassischen Altersdermatosen mit der Gruppe der bullösen Autoimmunerkrankungen, den Non-Melanoma Skin Cancers (NMSC) oder auch dem asteatotischem Ekzem zeigen sich neue Herausforderungen durch eine in der Breite älter werdenden Gesellschaft. Ein sich zügig entwickelndes und spannendes Gebiet ist das Verständnis der Immunseneszenz, der immunologischen Veränderungen im Zuge des Alterns. Ein weiteres großes Thema für uns Derma-

\title{
Dermatologie im Alter
}

tologen sind zudem Arzneimittelnebenwirkungen und -interaktionen einer polypharmazierten Gesellschaft.

Im ausgewählten Übersichtsartikel «pH of the Skin: Issues and Challenges» aus Current Problems in Dermatology diskutieren Rippke et al. die Bedeutung des genuin sauren Hautmilieus für die Kolonisation der Haut und zeigen, wie Veränderungen des pH-Werts und eine Veränderung der bakteriellen Flora Infektionen begünstigen. Dies hat Einfluss auf die Entstehung von Mykosen bis hin zur Abheilung eines Ulcus cruris. Ursächlich werden Einflussfaktoren wie ein Diabetes mellitus oder auch eine ungünstige Hautpflege einbezogen.

Im Wissenstransfer haben wir ebenfalls einen Focus auf präferenziell im Alter auftretende Erkrankungen gelegt und Themen wie das Lentigo-maligna-Melanom, das UIcus cruris hypertonicum martorell (ja, das gibt es wirklich) oder auch das chronische Handekzem ausgewählt.

Eine Veränderung der aktuell herrschenden Situation wird mit Sicherheit die Teledermatologie mit sich bringen. Frau Dr. Kirsten hat zu diesem Thema einen vergleichenden Artikel über die aktuell verfügbaren Optionen kommentiert und Vor- als auch Nachteile der Optionen zusammengestellt.

Gleich zwei Kommentierungen haben wir zu einer Arbeit, welche die Bedeutung von bestehenden Depressionen für den Therapieerfolg untersucht hat. Auch zu diesem Thema herrscht Handlungsbedarf. Bei Hauterkrankungen besteht häufig psychische Komorbidiät, teils auch als Causa für die Hauterkrankung, die, wie am Beispiel der Psoriasis gezeigt, einen negativen Einfluss auf den Therapieerfolg darstellen kann. Das sollte speziell unter hochpreisigen Medikationen wenn möglich vermieden werden. Da auch die Patienten in der Dermatologie im Schnitt den älteren Teil der Bevölkerung wiederspiegeln, hoffen wir Ihnen mit der getroffenen Auswahl Anregungen und vielleicht auch Hilfestellungen für die Praxis oder die Klinik geben zu können und wünschen viel Freude beim Lesen.

Mit vielen kollegialen Wünschen

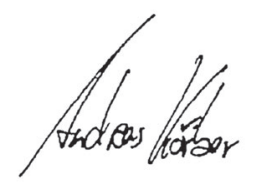

Ihr Prof. Dr. Andreas Körber

\section{KARGER}

Fax +4976145207 14 information@karger.com www.karger.com
() 2019 S. Karger GmbH, Freiburg
Prof. Dr. Andreas Körber

Gemeinschaftspraxis Dr. Cesko \& Prof. Dr. Körber

Rüttenscheider Str. 143, 45147 Essen, Deutschland Andreas.koerber@uk-essen.de 